\title{
Serviço de visão subnormal do Instituto Brasileiro de Oftalmologia e Prevenção da Cegiueira (IBOPC): análise dos pacientes atendidos no 1 ano do departamento (2004)
}

\author{
Low vision service at the Instituto Brasileiro de Oftalmologia $e$ \\ Prevenção da Cegueira (IBOPC): analysis of the patients examined \\ on the first year of the department (2004)
}

Ana Maria Tavares da Costa Pinto Silva ${ }^{1}$ Marta Hercog' Batista Rebelo de Matos ${ }^{2}$ Humberto de Castro Lima ${ }^{3}$
Trabalho realizado no Instituto Brasileiro de Oftalmologia e Prevenção da Cegueira - IBOPC - Salvador - BA.

Residente do $3^{\circ}$ ano de Oftalmologia do Instituto Brasileiro de Oftalmologia e Prevenção da Cegueira - IBOPCSalvador (BA) - Brasil

${ }^{2}$ Chefe do Departamento de Visão Subnormal e Preceptora de Estrabismo do IBOPC - Salvador (BA) - Brasil. Ex-Professor Titular da Escola Bahiana de Medicina e Saúde Pública. Diplomado Oftalmologista pelo "American Board of Ophthalmology" da América do Norte e Cirurgião-Oculista pelo New York Eye and Ear Infirmary. Livre docente da Faculdade de Medicina da Universidade Federal do Rio de Janeiro - UFRJ - Rio de Janeiro (RJ) - Brasil.

Endereço para correspondência: Marta Hercog B.R Matos. Rua Tenente Fernando Tuy, 139/102 - Salvador (BA) CEP 41810-780

E-mail: hmrdematos@uol.com.br

Recebido para publicação em 02.02.2006

Última versão recebida em 17.09.2009

Aprovação em 22.06.2010

\begin{tabular}{l} 
RESUMO \\
\hline Objetivo: Traçaro perfil dos pacientes portadores de baixa visão atendidos \\
no serviço de visão subnormal do Instituto Brasileiro de Oftalmologia e \\
Prevenção da Cegueira (IBOPC). Métodos: Realizou-se um estudo trans- \\
versal no qual foram revisados 82 prontuários de pacientes com baixa \\
visão, atendidos pelo Serviço Unnico de Saúde (SUS) no primeiro ano do \\
departamento de Visão Subnormal do Instituto Brasileiro de Oftalmologia \\
e Prevenção da Cegueira em 2004. Os dados analisados foram: sexo, faixa \\
etária, etiologia, acuidade visual inicial e final (pós-refração) para longe e \\
perto, come sem auxílio óptico e recurso óptico indicado. Resultados: Dos \\
82 pacientes avaliados, 11 (13,4\%) foram excluídos do trabalho por não \\
apresentarem visão subnormal. Dos 71 pacientes restantes, 32 (45\%) eram \\
menores de 20 anos. Quanto ao sexo, não houve diferença estatisticamente \\
significante (51\% eram mulheres e 49\% eram homens). A etiologia mais \\
frequente em crianças e adolescentes foi oglaucoma congênito (15,6\%). Em \\
pacientes de 20 a 39anos prevaleceu a toxoplasmose ocular (21,1\%). Entre \\
os pacientes de 40 a 59 anos, a retinose pigmentar foi a patologia mais \\
frequente (19\%). Nos idosos, o glaucoma foi a patologia mais encontrada \\
(40\%). Trinta e três pacientes (40,2\%) tinham acuidade visual entre 20/60 \\
e 20/160. O sistema telescópico foi o único auxílio óptico indicado para \\
longe (44\%) e os óculos foram os mais indicados para perto (54,5\%). \\
Conclusão: Existe uma alta prevalência de baixa visão em crianças e \\
adolescentes em países em desenvolvimento como o Brasil. Desta forma \\
o oftalmologista precisa criar programas preventivos, melhorar as condi- \\
ções de atendimento e atentar para o diagnóstico precoce e o tratamento \\
destes pacientes.
\end{tabular}

Descritores: Baixa visão/etiologia; Baixa visão/reabilitação; Baixa visão/classificação; Visão/epidemiologia; Acuidade visual; Cegueira/prevenção \& controle

\section{INTRODUÇÃ̃O}

A décima revisão da classificação internacional de doenças e problemas relacionados à saúde (CID-10) define que uma pessoa tem visão subnormal (VSN), ou baixa visão, quando sua acuidade visual corrigida no melhor olho é menor que 20/70 e maior ou igual a 20/400 e cegueira quando 
esses valores estão abaixo de 20/400 ${ }^{(1)}$. Segundo o relatório de consultoria da Organização Mundial de Saúde-OMS, conceitua-se como portadora de VSN aquela pessoa que possui um comprometimento do funcionamento visual mesmo após tratamento e/ou correção dos erros refracionais comuns, e com acuidade visual inferior a 20/60 até percepção de luz ou campo visual inferior a $10^{\circ}$ no ponto de fixação, mas que utiliza ou é potencialmente capaz de utilizar a visão para o planejamento e execução de uma tarefa ${ }^{(2-3)}$. Esta definição não foi incorporada ao CID -10. Faye (1984) salientou que, do ponto de vista clínico, além da acuidade visual e do campo visual, o portador de VSN pode apresentar outras funções visuais afetadas tais como a percepção de cores, sensibilidade ao contraste, adaptação à luz e ao escuro, fusão e percepção visual $^{(4)}$.

A OMS estima que cerca de $1 \%$ da população mundial apresenta algum grau de deficiência visual, sendo que $90 \%$ destas encontram-se em países em desenvolvimento ${ }^{(2)}$. Em nosso país, todavia, a falta de dados precisos e significativos dificulta o dimensionamento do problema.

A maior funcionalidade visual é um dos principais objetivos do atendimento oftalmológico do paciente com VSN. O conhecimento das principais causas de baixa visão e do perfil dos pacientes com deficiência visual permite otimizar o atendimento dos mesmos nos serviços de oftalmologia e traçar estratégia para ações relacionadas à prevenção da cegueira.

O objetivo do presente estudo é traçar o perfil epidemiológico e oftalmológico dos pacientes com VSN que utilizam o nosso serviço.

\section{MÉTODOS}

Foi realizado um estudo transversal analisando prontuários de 82 pacientes do SUS (Sistema Único de Saúde) atendidos no departamento de VSN do Instituto Brasileiro de Oftalmologia e Prevenção da Cegueira (IBOPC). Foram considerados dados como sexo, faixa etária, etiologia, uso prévio de auxílio óptico, acuidade visual inicial e final (pós-refração) para perto e longe com e sem auxílio óptico e tipo de auxílio indicado.

Os pacientes atendidos no departamento de VSN foram encaminhados pelos ambulatórios das demais subespecialidades de oftalmologia com exame oftalmológico completo e diagnóstico definido.

No ambulatório de VSN o paciente é submetido à anamnese, refração, aferição da acuidade visual inicial e pós-refração para perto e longe e então são testados auxílios ópticos conforme a idade e necessidade requerida pelo paciente pesquisando, finalmente a acuidade final obtida com o auxílio. A avaliação foi realizada numa sala com iluminação de 807 lux e condição de iluminação em relação à potência da fonte e dimensões do ambiente de $80 \mathrm{~cd} / \mathrm{m}^{2}$, contendo cadeira, coluna, Greens, caixa de prova, armação de prova, foco com lâmpada incandescente de 60 watts, armação móvel com ilumi- nação da marca xenônio e tabelas ETDRS (Lighthouse, New York, cat. $\mathrm{n}^{\circ} \mathrm{C} 105$ ) e tabela Lea symbols (Good-Lite, New York, $n^{\circ}$ 250300). Foi realizada a refração estática e dinâmica através da retinoscopia. Nos casos de dificuldade de determinação do eixo do astigmatismo foi realizada a ceratoscopia. A refração subjetiva foi realizada com caixa de prova.

A acuidade visual dos pacientes envolvidos no trabalho foi aferida da seguinte forma:

- Tabela Lea symbols, para pacientes iletrados a uma distância de $75 \mathrm{~cm}, 1,5$ ou 3,0 m;

- Tabela ETDRS (Early Treatment Diabetic Retinopathy Study) para pacientes letrados colocadas a $50 \mathrm{~cm}, 1,0$, 2,0 ou 4,0 m.

Devido à idade ou a distúrbios cognitivos, alguns pacientes não informaram a visão pelas tabelas descritas acima. Nestes foram realizados campo visual de confrontação, avaliação da visão com luz e objetos de alto contraste, porém não foi possível quantificar a acuidade visual devido à falta das cartelas de Teller.

A acuidade visual para longe foi qualificada segundo a classificação da gravidade de comprometimento visual da OMS:

Grupo I: 20/30 a 20/60 - variação da visão normal.

Grupo II: <20/60 a 20/160 - perda leve.

Grupo III: 20/200 a 20/400 - perda moderada.

Grupo IV: 20/500 a 20/1000 - perda grave.

Grupo V: 20/1250 a percepção de luz - perda profunda.

Considerou-se sempre a acuidade visual do melhor olho com a melhor correção óptica obtida no departamento de VSN. Foram excluídos do trabalho os pacientes que apresentavam acuidade visual melhor ou igual a 20/60 no melhor olho corrigido.

A distribuição dos pacientes por faixa etária foi realizada considerando como criança e adolescentes aqueles entre 0 e 19 anos, o adulto jovem entre 20 e 39 anos, o adulto entre 40 e 59 anos e idosos com 60 anos ou mais.

Quanto à distribuição etiológica, foi considerada a causa primária da deficiência visual. Foi descrita mais de uma causa quando patologias distintas eram responsáveis pela baixa visão.

$\mathrm{Na}$ avaliação dos auxílios ópticos considerou-se dois grupos. Os recursos ópticos para longe: os sistemas telescópicos de $3 \mathrm{x}$ e de $4 \mathrm{x}$ (tipo Kepler), monocular, com foco ajustável, além do telessistema tipo Coil 2x (binocular, com foco ajustável). Estes auxílios eram indicados para crianças com queixa de dificuldade para enxergar o quadro negro, pacientes cujo objetivo era enxergar letreiro de ônibus e placas ou assistir televisão. Os auxílios ópticos para perto: lupa manual sem iluminação de 8 e 16 dioptrias, lupas de apoio sem iluminação e com iluminação (28 e 32 dioptrias), barras de leituras (1,5 e 2 dioptrias), óculos esferoprismáticos de +6 e 8 dioptrias prismáticas até +12 e 14 dioptrias prismáticas, óculos asféricos de +12 dioptrias a +28 dioptrias. As lupas foram indicadas principalmente para crianças, adolescentes e adultos jovens que não obtiam visão de perto satisfatória com a 
268 Serviço de visão subnormal do Instituto Brasileiro de Oftalmologia e Prevenção da Cegueira (IBOPC): análise dos pacientes atendidos no $1^{\circ}$ ano do departamento (2004)

aproximação e para adultos e idosos com defeitos importantes de campo visual sem tremores. Os óculos foram indicados principalmente para adultos e idosos com objetivo de leitura ou outra atividade de perto.

A maioria dos pacientes foi encaminhada para treinamento do auxílio no CAP (Centro de Auxílio Pedagógico ao Deficiente Visual) para posterior prescrição quando apresentavam uma boa resposta. Lá também são dadas orientações sobre os auxílios não ópticos (iluminação, contraste, ampliação), treinamento de atividade de vida diária e mobilidade. Apenas os pacientes para os quais só foi indicado óculos para leitura e a desempenharam bem pelo uso funcional do auxílio óptico, a uma distância mínima de $10 \mathrm{~cm}$, não foram encaminhados ao CAP, a menos que apresentasse dificuldade na orientação e mobilidade.

Os pacientes de 0 a 3 anos foram encaminhados para estimulação visual precoce.

\section{RESULTADOS}

De janeiro a novembro de 2004 foram atendidos no departamento de VSN 82 pacientes encaminhados pelos departamentos de outras subespecialidades da Oftalmologia. Após refração cuidadosa destes pacientes, observou-se que 11 apresentavam acuidade visual no melhor olho maior ou igual a 20/60 e então não eram portadores de VSN, mostrando a grande importância de uma refração cautelosa.

Quanto ao sexo, 36 pacientes (51\%) eram do sexo feminino e $35(49 \%)$ do masculino.

Quanto à faixa etária, os 71 pacientes com visão subnormal tinham entre 6 meses e 86 anos sendo que, 32 (45\%) eram menores de 19 anos, 14 (20\%) tinham entre 20 e 39 anos, 15 (21\%) tinham de 40 a 59 anos e $10(14 \%)$ eram maiores ou iguais a 60 anos (Figura 1).

Quanto à etiologia, observou-se que dentre os pacientes com visão subnormal, as patologias mais prevalentes foram coriorretinite por toxoplasmose $(10,4 \%)$, glaucoma $(9,1 \%)$, alta miopia $(9,1 \%)$, retinose pigmentar $(7,8 \%)$ e albinismo oculocutâneo $(7,8 \%)$ (Figura 2).

Analisando as etiologias nas diferentes faixas etárias observou-se que em menores de 20 anos o glaucoma congênito

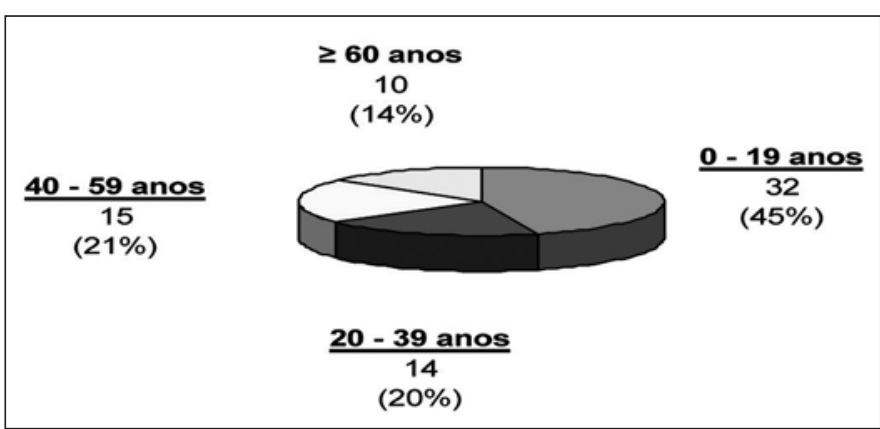

Figura 1 - Distribuição dos pacientes por faixa etária foi a patologia mais prevalente sendo responsável por $15,6 \%$ dos casos, seguido pela toxoplasmose ocular (12,5\%) e albinismo (12,5\%), atrofia óptica (12,5\%) e nistagmo (12,5\%). De 20 a 39 anos houve maior prevalência de toxoplasmose ocular $(21,1 \%)$ seguida por retinose pigmentar $(15,8 \%)$ e maculopatia (15,8\%). Entre os pacientes de 40 a 59 anos prevaleceu a retinose pigmentar $(19 \%)$ seguido pelo glaucoma (13\%), albinismo (13\%) e a retinopatia diabética (13\%). Nos idosos, pacientes de 60 anos ou mais, o glaucoma foi a patologia mais frequente $(40 \%)$ seguido pela DMRI (30\%).

Foi avaliada a acuidade visual de todos pacientes encaminhados ao departamento de VSN (82 pacientes) e distribuídas de acordo com a classificação de comprometimento visual da OMS. Desta forma o grupo I obteve 11 casos $(23,4 \%)$; o grupo II, 33 casos (40,2\%); o grupo III, 19 casos (23,2\%); o grupo IV, 8 casos $(9,8 \%)$ e o grupo V obteve 3 casos $(3,6 \%)$, totalizando $90,2 \%$ dos pacientes. Os demais ( 8 pacientes) não informaram a acuidade: 1 era surdo-mudo e não colaborou com o teste de pareamento, 3 tinham distúrbios cognitivos e 4 eram menores de 3 anos.

O telessistema foi o único auxílio óptico prescrito para longe, sendo indicado para 35 pacientes (44\%). Destes, 15 pacientes $(42,85 \%)$ tinham entre 0 e 19 anos; 16 pacientes $(45,72 \%)$, entre 20 e 39 anos e 4 pacientes $(11,42 \%)$, entre 40 e 59 anos. Os auxílios ópticos para perto foram indicados para 44 pacientes $(56 \%)$. As lentes esféricas foram indicadas para 15 pacientes $(33,6 \%)$. Um paciente $(6,66 \%)$ tinha entre 0 e 19 anos; 3 pacientes (20\%), entre 20 e 39 anos; 5 pacientes $(33,33 \%)$, entre 40 e 59 anos e 6 pacientes (40\%), maiores que 60 anos. As lentes esferoprismáticas foram prescritas para 7 $(15,08 \%)$, destes, 2 pacientes $(28,57 \%)$ apresentavam idade entre 40 a 59 anos e 5 pacientes $(71,42 \%)$, maiores que 60 anos. As lentes asféricas foram indicadas para apenas $2(4,5 \%)$ pacientes acima de 60 anos; a lupa de mesa foi indicada para 17 pacientes $(38,6 \%)$. Nove pacientes $(52,94 \%)$ entre 0 e 19 anos, 4 pacientes $(23,52 \%)$ entre 20 e 39 anos, 3 pacientes $(17,64 \%)$ entre 40 e 59 anos e 1 paciente $(5,88 \%)$ acima de 60 anos. A lupa de mão foi prescrita para $3(6,8 \%)$ - um $(33,33 \%)$ tinha entre 20 e 39 anos e $2(66,66 \%)$, entre 40 e 59 anos.

\section{DISCUSSÃO}

O IBOPC é um centro de referência para a saúde ocular da população carente da Bahia. É uma instituição ligada à Escola Bahiana de Medicina e à Fundação Bahiana para o Desenvolvimento das Ciências promovendo o ensino e a pesquisa. O departamento de VSN do IBOPC foi fundado em janeiro de 2004 com o objetivo de atender as necessidades dos baianos carentes com deficiência visual.

Quanto ao sexo, 51\% dos pacientes com VSN eram mulheres e $49 \%$ eram homens, o que está compatível com a maioria dos trabalhos publicados nos quais não houve diferença estatisticamente significativa em relação ao $\operatorname{sexo}^{(2,5)}$.

Quanto à faixa etária, $45 \%$ dos pacientes com VSN tinham menos de 20 anos e $65 \%$ são menores de 40 anos. Esta maior 


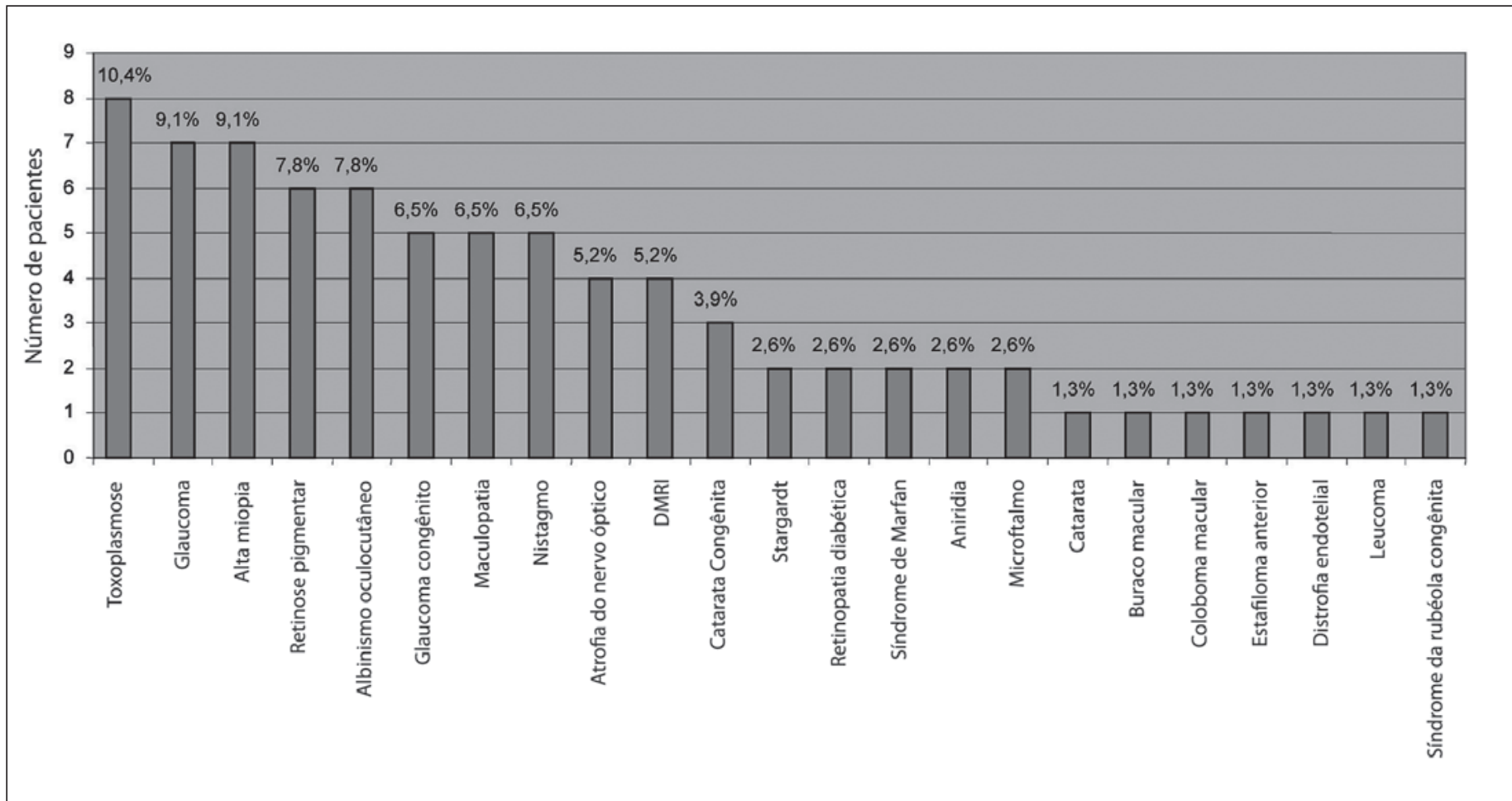

Figura 2 - Distribuição dos pacientes por etiologia da baixa visão

prevalência de deficiência visual em crianças, adolescentes e adultos jovens também é observada em outros estudos realizados em países em desenvolvimento e inclusive no Bra$\mathrm{sil}^{(2,6-7)}$. Alguns autores em 1995, constataram que $75 \%$ dos pacientes atendidos no departamento de VSN de um serviço público eram crianças e adultos jovens $(0-40 \text { anos })^{(6)}$. Nos países desenvolvidos, a maioria dos portadores de baixa visão são os idosos ${ }^{(8-9)}$.

Quanto à etiologia da baixa visão, a mais prevalente foi coriorretinite por toxoplasmose $(10,4 \%)$ o que também foi encontrado por alguns autores em $1998^{(2)}$. As principais causas de VSN observadas por alguns autores em 1995 foram catarata congênita (13\%), retinose pigmentar (9\%), atrofia de nervo óptico $(8 \%)$ e alta miopia $(7 \%)^{(6)}$. Outros autores em 1991, detectaram que as causas mais comuns de baixa visão em crianças entre 0 e 5 anos foram: coriorretinite por toxoplasmose $(30 \%)$, glaucoma congênito $(12 \%)$ e catarata congênita $(12 \%)^{(10)}$. Lucas et al., constataram que as principais causas de VSN entre 0 e 19 anos foi a catarata congênita ${ }^{(7)}$. Em países desenvolvidos atrofia do nervo óptico e retinopatia da prematuridade são responsáveis pela maior parte das deficiências visuais na infância ${ }^{(9)}$. No presente estudo, foi observado que em crianças e adolescentes as principais causas de baixa visão foi o glaucoma congênito $(15,6 \%)$, seguido pela toxoplasmose ocular (12,5\%), atrofia óptica (12,5\%), albinismo $(12,5 \%)$ e nistagmo (12,5\%). Entre nossos pacientes de 20 a 39 anos houve maior prevalência de toxoplasmose ocular $(21,1 \%)$, seguida por retinose pigmentar $(15,8 \%)$ e maculo- patia $(15,8 \%)$ e entre os pacientes de 40 a 59 anos prevaleceram a retinose pigmentar (19\%), seguido pelo glaucoma (13\%), o albinismo (13\%) e a retinopatia diabética (13\%). Lucas et al., constatou que as principais causas de VSN entre 20 e 59 anos foi a retinose pigmentar ${ }^{(7)}$. No nosso estudo, o glaucoma foi a patologia mais frequente (40\%) entre os idosos, o que também foi observado por Lucas et al. ${ }^{(7)}$. Por outro lado, outros trabalhos observaram que em pacientes idosos a degeneração macular relacionada à idade (DMRI) foi responsável pela maioria dos casos de deficiência visual ${ }^{(2,5)}$. Um estudo que comparou pacientes maiores de 60 anos, atendidos em serviços público e privado, encontrou o glaucoma e a degeneração macular relacionada à idade como principais causas de baixa visão nos serviços público e privado respectivamen$\mathrm{te}^{(11)}$. Em países desenvolvidos a principal causa de VSN em idosos é a DMRI ${ }^{(8-9)}$.

O telessistema foi o único auxílio óptico prescrito para longe, sendo indicadas para $44 \%$ dos pacientes. Esta grande porcentagem de prescrição de telessistemas provavelmente se deve ao fato de que grande parte dos pacientes do estudo era composta de crianças e adolescentes em idade escolar, e de adultos jovens com objetivo de atividades à distância como ler letreiro de ônibus, por exemplo. Os auxílios ópticos para perto foram mais indicados, o que está de acordo com outros trabalhos publicados nos quais esses auxílios foram os mais aceitos e, portanto, mais prescritos, principalmente os ócu$\operatorname{los}^{(2,5,7,11)}$. Os auxílios para perto foram indicados para 44 pacientes $(55 \%)$. As lentes esféricas foram indicadas para 15 
270 Serviço de visão subnormal do Instituto Brasileiro de Oftalmologia e Prevenção da Cegueira (IBOPC): análise dos pacientes atendidos no $1^{\circ}$ ano do departamento (2004)

pacientes $(34,1 \%)$, as lentes esferoprismáticas para $7(15,9 \%)$ e as lentes asféricas foram indicadas para apenas 2 pacientes $(4,5 \%)$; a lupa de mesa foi indicada para 17 pacientes $(38,6 \%) \mathrm{e}$ a lupa de mão para $3(6,8 \%)$. Nosso trabalho difere do de Castro no qual os auxílios ópticos mais prescritos foram telessistema $(30,2 \%)$, lentes asféricas $(28,2 \%)$, lentes esferoprismáticas $(17,8 \%)$ e lentes esféricas $(10 \%)$ e os menos prescritos foram telessistema com acoplagem (7,5\%), lupa manual (4\%), lupa de apoio $(1,1 \%)$ e lentes filtrantes $(0,8 \%)^{(12)}$.

Quanto à acuidade visual para longe, observamos que a maioria dos pacientes $(40,2 \%)$ apresentava perda moderada de visão o que também foi observado por alguns autores ${ }^{(5,7,11)}$.

\section{CONCLUSÃO}

A importância deste trabalho se dá pela carência de publicações sobre o tema não só na Bahia, mas também no Brasil. Chama a atenção dos oftalmologistas sobre a importância do diagnóstico e tratamento precoces dos pacientes com deficiência visual principalmente na infância. O tratamento destes pacientes busca a maior funcionalidade visual permitindo a inclusão dos mesmos na sociedade. Este estudo se propõe a conhecer o paciente com visão subnormal com o objetivo de viabilizar seu atendimento mais adequado e efetivo nos centros de oftalmologia.

\section{ABSTRACT}

Purpose: To outline low vision (LV) patients profile of the low vision department of the Instituto Brasileiro de Oftalmologia e Prevenção da Cegueira (IBOPC). Methods: Transversal study of 82 medical charts from the low vision service at the Instituto Brasileiro de Oftalmologia e Prevenção da Ceguei$r a$ of the first year of the department - 2004, considering age, gender, etiology, distance and near visual acuity with and without optical aids and prescription assistance. Results: Of the 82 available patients, 11 (13.4\%) were excluded of the study because they did not present low vision. Of the 71 patients, 32 $(45 \%)$ were younger than 20 years old. Regarding the gender, there was not a significant difference $(51 \%$ female and $49 \%$ male). The most frequent etiology in infants and adolescents was congenital glaucoma and in patients aged between 20-39 years was ocular toxoplasmosis (21.1\%). In patients with 40-59 years old, pigmentary retinosis was the most frequently pathology (19\%). In elderly people it was glaucoma (49\%). Thirty-three patients $(40.2 \%)$ had visual acuity between $20 / 60$ and 20/160. The telescopic system was the only optical aid indicated for distance (44\%) and glasses were the most indicated for near (54.5\%). Conclusion: There is a high prevalence of low vision in infants and adolescents that live in developing countries like Brazil. Therefore, ophthalmologists need to be aware to promote early diagnosis and treatment to these patients, creating preventive programs and better assistance conditions.

Keywords: Vision, low/etiology; Vision, low/rehabilitation; Vision, low/classification; Vision, low/epidemiology; Visual acuity; Blindness/prevention \& control

\section{REFERÊNCIAS}

1. Sampaio MW, Haddad MAO, Kara-José N. Auxílios para baixa visão. São Paulo: Laramara; 2001.

2. Reis PAC, Campos CMC, Fernandes LC. Características da população portadora de visão subnormal do Hospital São Geraldo: um estudo retrospectivo de 435 casos. Rev Bras Oftalmol. 1998;5(4)7:287-94.

3. Moya STF, Oliveira AA, Fernandes LC. Retinose pigmentar e visão subnormal: adaptação aos auxílios ópticos para perto e correlação com funções visuais Rev Bras Oftalmol. 2003;63(9):679-88.

4. Faye EE. Clinical low vision. Boston: Little Brown; 1984.

5. De Lucca ES, Carrari MI, Laçava AC, Kubo SLU, Kinoshita MH. Características da população de visão subnormal em clinica oftalmológica não universitária. Rev Bras Oftamol. 1999;58(11):879-87.

6. Leal DB, Tavares SS, Ventura LO, Florêncio T. Atendimento a portadores de visão subnormal: estudo retrospectivo de 317 casos. Arq Bras Oftalmol. 1995; 58(6):439-42.

7. Lucas MB, Leal DB, Tavares SS, Barros EA, Aranha ST. Condutas reabilitacionais em pacientes com baixa visão. Arq Bras Oftalmol. 2003;66(1):77-82.

8. De Winter LJ, Hoyng CB, Froeling PG, Meulendijks CF, Van der Wilt GJ. Prevalence of remediable disability due to low vision among institutionalized elderly people. Gerontology. 2004;50(2):96-101.

9. Pascolini D, Mariotti SP, Pokharel GP, Pararajasegaram R. 2002 Global up date of available data on visual impairment: a compilation of population - based prevalence studies. Ophthalmic Epidemiol. 2004;11(2):67-115.

10. Tartarella MB, Nakano K, Castro CTM, Martins APM. Visão subnormal em crianças. Arq Bras Oftalmol. 1991;54(5):221-4.

11. Lucas MB, Leal DB, Tavares SS, Diniz JR, Cantanhede MI. Baixa visão em idosos - estudo comparativo entre um serviço público e um serviço privado da cidade de Recife. Rev Bras Oftalmol. 2003;62(2):138-43.

12. Castro DDM. Visão subnormal. Rio de Janeiro: Cultura Médica; 1994 